\title{
Recent Developments in Quantum Energy Inequalities
}

\author{
Christopher J. Fewster \\ Department of Mathematics, University of York, \\ Heslington, York YO10 5DD, United Kingdom. \\ E-mail: cjf3@york.ac.uk
}

\begin{abstract}
Two recent developments in the theory of Quantum Energy Inequalities (QEIs) are reported: first, an absolute QEI in curved spacetimes; second, the use of local covariance in combination with QEIs to obtain a priori bounds on the renormalized stress tensor.

Keywords: Quantum field theory in curved spacetime, Quantum energy inequalities
\end{abstract}

\section{Introduction}

In General Relativity, the stress tensor $T_{a b}$ is often assumed to obey the Weak Energy Condition (WEC) that $T_{a b} u^{a} u^{b}$ should be everywhere nonnegative for all timelike $u^{a}$. Although the classical energy conditions are violated by quantum fields, there are remnants of these conditions, called Quantum Energy Inequalities (QEIs) [or, more briefly, Quantum Inequalities (QIs)] which apply to suitable averages

$$
\langle\mathrm{T}(\mathrm{f})\rangle_{\omega}:=\int\left\langle T_{a b}\right\rangle_{\omega} f^{a b} d \mathrm{vol}
$$

of the expectation value of the renormalized stress-energy tensor in state $\omega$. (See Refs. 1-3 for recent reviews and references.) There are two types of QEIs: absolute QEIs (AQEIs), which take the form

$$
\langle\mathrm{T}(\mathrm{f})\rangle_{\omega} \geq-\mathcal{Q}(\mathrm{f}) \text { for all (physically reasonable) states } \omega,
$$

and difference QEIs (DQEIs), which take the form

$$
\langle\mathrm{T}(\mathrm{f})\rangle_{\omega}-\langle\mathrm{T}(\mathrm{f})\rangle_{\omega_{0}} \geq-\mathcal{Q}\left(\mathrm{f}, \omega_{0}\right) \text { for all (physically reasonable) states } \omega,
$$

where $\omega_{0}$ is a reference state. As a concrete example, the massless scalar field in four-dimensional Minkowski space obeys the AQEI

$$
\int\left\langle T_{00}(t, 0)\right\rangle_{\omega}|g(t)|^{2} d t \geq-\frac{1}{16 \pi^{2}} \int\left|g^{\prime \prime}(t)\right|^{2} d t
$$

for all Hadamard states $\omega$ and all smooth, real-valued functions $g$ vanishing outside a compact set. A simple consequence ${ }^{4}$ is that if $\left\langle T_{00}\right\rangle_{\omega}(t, 0)<\mathcal{E}$ for $0 \leq t \leq \tau$, then $\mathcal{E} \geq-C / \tau^{4}$, where $C=3.16 \ldots$ in units where $\hbar=c=1$. This illustrates the close links between the QEIs and intuition based on the uncertainty principle.

\section{Absolute Quantum Energy Inequalities}

In curved spacetimes, the most general results known are difference QEIs (e.g., Ref. 5 for the scalar field). This hampers attempts to use QEIs to constrain exotic spacetimes ${ }^{6-8}$ because one does not typically have explicit access to a reference 
state $\omega_{0}$. The typical approach is to use the equivalence principle to argue that Minkowski space QEIs such as Eq. (1) apply on sufficiently small scales. Here, we describe recent work with C.J. Smith, ${ }^{9}$ in which the first explicit AQEIs in general four-dimensional curved spacetimes are obtained.

Consider the quantized minimally coupled Klein-Gordon field with mass $m \geq 0$ in four spacetime dimensions. In state $\omega$, the expected renormalized stress tensor is

$$
\left\langle T_{\mu \nu}\right\rangle_{\omega}(x)=\left[P_{(\mu \nu)}\left(\Lambda_{\omega}-H_{k}\right)\right](x, x)-Q(x) \eta_{\mu \nu}+C_{\mu \nu}(x)
$$

when expressed in terms of a tetrad, where $\Lambda_{\omega}\left(x, x^{\prime}\right)=\left\langle\phi(x) \phi\left(x^{\prime}\right)\right\rangle_{\omega}$ is the two-point function, the $P_{\mu \nu}$ are differential operators given by

$$
P_{\mu \nu}=\nabla_{\mu} \otimes \nabla_{\nu}-\frac{1}{2} \eta_{\mu \nu} \eta^{\alpha \beta} \nabla_{\alpha} \otimes \nabla_{\beta}+\frac{1}{2} m^{2} \eta_{\mu \nu}
$$

and $H_{k}\left(x, x^{\prime}\right)$ is the partial Hadamard $\operatorname{sum}^{10}$

$$
H_{k}=\frac{\Delta^{1 / 2}}{4 \pi^{2} \sigma_{+}}+\sum_{\ell=0}^{k}\left[v_{\ell} \sigma^{\ell} \log \sigma_{+}+w_{\ell} \sigma^{\ell}\right] .
$$

In Eq. (2), the term $Q(x)$ is added to ensure conservation, and $C_{\mu \nu}(x)$ is a conserved, local curvature term. The definition is independent of $k$ provided it is at least 2 . Our AQEI may now be stated as follows:

Theorem 2.1. Let $\mathcal{O}$ be an open region in a globally hyperbolic spacetime such that the $H_{k}$ exist on $\mathcal{O} \times \mathcal{O}$. Let $\gamma: I \rightarrow \mathcal{O}$ be a proper-time parameterisation of a smooth, future-directed timelike curve, where $I$ is an open interval of $\mathbb{R}$, and suppose $e_{\mu}^{a}$ is a tetrad on $\mathcal{O}$ which is invariant under Fermi-Walker transport along $\gamma$, where it obeys $\left.e_{0}^{a}\right|_{\gamma}=\dot{\gamma}^{a}$. Then the AQEI

$$
\int_{\gamma}\left\langle T_{a b} \dot{\gamma}^{a} \dot{\gamma}^{b}\right\rangle_{\omega} g(\tau)^{2} d \tau \geq \int_{\gamma}\left(C_{a b} \dot{\gamma}^{a} \dot{\gamma}^{b}-Q\right) g(\tau)^{2} d \tau-\frac{1}{\pi} \int_{0}^{\infty} \widehat{F_{k}}(-\alpha, \alpha) d \alpha
$$

holds for any Hadamard state $\omega$, any $g \in C_{0}^{\infty}(I ; \mathbb{R})$ and any $k \geq 5$, where

$$
F_{k}\left(\tau, \tau^{\prime}\right)=g(\tau) g\left(\tau^{\prime}\right)\left(P_{00} \widetilde{H}_{k}\right)\left(\gamma(\tau), \gamma\left(\tau^{\prime}\right)\right)
$$

and $\widetilde{H}_{k}\left(x, x^{\prime}\right)=\frac{1}{2}\left[H_{k}\left(x, x^{\prime}\right)+H_{k}\left(x^{\prime}, x\right)+i E\left(x, x^{\prime}\right)\right]$, with $E$ denoting the advancedminus-retarded fundamental bisolution. (We write $\widehat{F}(k)=\int d^{n} x e^{i k \cdot x} F(x)$.)

This bound is similar in form to an older DQEI, ${ }^{5}$ in which the terms involving $C_{a b}$ and $Q$ are absent and $\widetilde{H}_{k}$ is replaced by the two-point function $\Lambda_{\omega_{0}}$ of a reference state. The proofs differ in that $\Lambda_{\omega}-\Lambda_{\omega_{0}}$ is smooth, while $\Lambda_{\omega}-\widetilde{H}_{k}$ is only $C^{k}$, necessitating a more refined analysis using Sobolev wave-front sets. Similar results may be obtained for averages over worldvolumes and other timelike spacetime submanifolds. Note that the AQEI bound is independent of the state $\omega$ and is defined in terms of local geometrically constructed objects such as the Hadamard series coefficients (the result is independent of the particular choice of $e_{\mu}^{a}$ ).

As the support of $g$ shrinks, the $\sigma_{+}^{-1}$ contribution to $\widetilde{H}_{k}$ dominates: the bound becomes Minkowskian. A more careful analysis of this limit, giving precise estimates, would justify the arguments used to apply QEIs to constrain exotic spacetimes. 


\section{QEIs and local covariance}

A general DQEI on spacetime $\mathcal{M}$ has the schematic form

$$
\left\langle\mathrm{T}_{\mathcal{M}}(\mathrm{f})\right\rangle_{\omega}-\left\langle\mathrm{T}_{\mathcal{M}}(\mathrm{f})\right\rangle_{\omega_{0}} \geq-Q_{\mathcal{M}}\left(\mathrm{f}, \omega_{0}\right)
$$

(using $\mathcal{M}$ to denote the underlying manifold, its metric and choices of (time)orientation). If an isometry $\psi$ embeds a globally hyperbolic spacetime $\mathcal{N}$ as a globally hyperbolic subset of a globally hyperbolic spacetime $\mathcal{M}$, then we may pull back a state $\omega$ on $\mathcal{M}$ to a state $\psi^{*} \omega$ on $\mathcal{N}$ so that $\left\langle\mathrm{T}_{\mathcal{N}}(\mathrm{f})\right\rangle_{\psi^{*} \omega}=\left\langle\mathrm{T}_{\mathcal{M}}\left(\psi_{*} \mathrm{f}\right)\right\rangle_{\omega}$, where $\psi_{*} \mathrm{f}$ is the push-forward of $\mathrm{f}$ from $\mathcal{M}$ to $\mathcal{N}$. This relation asserts that the stress-energy tensor is covariantly defined. ${ }^{11}$ Certain DQEIs are also covariant, i.e.,

$$
Q_{\mathcal{N}}\left(\mathrm{f}, \psi^{*} \omega_{0}\right)=Q_{\mathcal{M}}\left(\psi_{*} f, \omega_{0}\right),
$$

and this permits us to use QEIs on $\mathcal{M}$ to constrain energy densities on $\mathcal{N}$. ${ }^{4,12,13}$ As a simple application, ${ }^{4}$ suppose a stationary spacetime $\mathcal{N}$ contains a stationary timelike geodesic segment $\gamma$ of proper duration $\tau_{0}$, which may be enclosed in a flat simply connected open globally hyperbolic subset $\mathcal{N}^{\prime}$ of $\mathcal{N}$. Then $\mathcal{N}^{\prime}$ is isometric to a globally hyperbolic subset of Minkowksi space, and we may apply the Minkowski QEIs along $\gamma$, to obtain an a priori bound

$$
\left\langle T_{a b} \dot{\gamma}^{a} \dot{\gamma}^{b}\right\rangle_{\omega_{0}} \geq-\frac{C \dot{\gamma}^{a} \dot{\gamma}_{a}}{\tau_{0}^{4}} \quad C=3.16 \ldots,
$$

on the energy density on $\gamma$ of the ground state $\omega_{0}$ of the Klein-Gordon field on $\mathcal{N}$. See Refs. 4,12 for other examples; the same idea can be used to prove the averaged null energy condition for null geodesics with suitable flat neighborhoods. ${ }^{14}$

\section{References}

1. L. H. Ford, in 100 Years of Relativity - Space-time Structure: Einstein and Beyond (World Scientific, Singapore, 2006) gr-qc/0504096.

2. T. A. Roman, in Proceedings of the Tenth Marcel Grossmann Meeting on General Relativity gr-qc/0409090.

3. C. J. Fewster, in XIVth International Congress on Mathematical Physics (World Scientific, Singapore, 2005); Expanded and updated version: math-ph/0501073.

4. C. J. Fewster and M. J. Pfenning, J. Math. Phys. 47, 082303 (2006).

5. C. J. Fewster, Class. Quantum Grav. 17, 1897 (2000).

6. L. H. Ford and T. A. Roman, Phys. Rev. D 53, 5496 (1996).

7. M. J. Pfenning and L. H. Ford, Class. Quantum Grav. 14, 1743 (1997).

8. C. J. Fewster and T. A. Roman, Phys. Rev. D 72, 044023 (2005).

9. C. J. Fewster and C. J. Smith, in preparation.

10. R. M. Wald, Quantum Field Theory in Curved Spacetime and Black Hole Thermodynamics (University of Chicago Press, Chicago, 1994).

11. R. Brunetti, K. Fredenhagen, and R. Verch, Commun. Math. Phys. 237, 31 (2003).

12. P. Marecki, Phys. Rev. D 73, 124009 (2006).

13. C. J. Fewster, math-ph/0611058.

14. C. J. Fewster, K. Olum and M. J. Pfenning, gr-qc/0609007. 